\title{
Agremiación Y ACCIÓN JUIVENIL EN ESPACIOS educativos de Córdoba, Argentina
}

\author{
Youth Action unIONIZATION AND EDUCATIONAL SPACES OF CORDOBA, ARGENTINA
}

Por: Mgtr. Gabriela Beatriz Rotondi*

*ROTONDI, GABRIELA

Magíster de la Uni-

versidad Nacional de

Córdoba. Argentina.

Filiación Institucional: Pro-

fesora Titular de la cátedra

Teoría, Espacios y Estra-

tegias de Intervención IV

- Institucional. Director

de la Investigación: Centros

de Estudiantes: Procesos de

institucionalización y apor-

te a la ciudadanía. Institu-

ción de referencia: Escuela

de Trabajo Social, Univer-

sidad Nacional de Córdoba.

Correo Electrónico: gabrie-

larotondi@hot not
Artículo recibido: Abril 27 de 2011. Artículo aprobado: Noviembre 26 de 2011

\section{RESUMEN}

El estudio de los derechos gremiales de los estudiantes secundarios en las escuelas públicas ha sido una temática escasamente indagada en nuestro medio. Las dificultades organizativas se encuentran relacionadas con las trayectorias estudiantiles en las instancias organizativas como los centros de estudiantes, las estructuras de poder institucional y normativo instaladas en las escuelas, y las particulares condiciones de los escenarios escolares. El juego de los diversos sujetos presentes en la institución se complejiza por la dinámica y ejercicio del poder en la escena institucional escolar. Al respecto nos preguntamos: ¿Qué parámetros organizativos se plantean respecto de la participación ciudadana de los jóvenes estudiantes? ¿Qué viabilidad plantean los gérmenes organizativos de estudiantes secundarios en los formatos actuales para la agremiación juvenil? Los derechos ciudadanos sean civiles, políticos, sociales gremiales o reproductivos, permanecen condicionados en términos estructurales, por la lógicas de organización social tanto como por los espacios sociales e institucionales, incluida la escuela. En tal sentido, la investigación que planteamos pretende hacer un aporte al estudio de las particularidades de los procesos de institucionalización de centros de estudiantes en escuelas medias y su relación con la construcción de representaciones sociales de los jóvenes en torno a la ciudadanía.

Palabras clave: Agremiación juvenil - ciudadanía.

\section{ABSTRACT}

The study of trade union rights of high school students in public schools has been a subject scarcely investigated in our academic environment. Organizational difficulties are related to student trajectories in organizational groups such as student centers, institutional power structures and policies installed in schools, and the particular conditions of school settings. The role of the people present in these institutions becomes more complex due to the dynamics and rules in this institutionalized school setting. In this regard we ask: -What organizational parameters arise regarding the participation of young students?; -What organizational viability does the conforming nucleus of high school students are able to offer in the current standards for youth unions? Citizens' rights either civil, political, social or reproductive associations, remain controlled, in structural terms, by the logic of social organization as well as by social and institutional spaces, including schools. In this regard, this research proposes a contribution to the study of the peculiarities of the process of institutionalization of student centers at middle schools and examines its relationship with the construction of social representations of young people in terms of citizenship.

Key words: Teenager unions - Citizenship.
Artículo de investigación científica y tecnológica según clasificación Colciencias. 


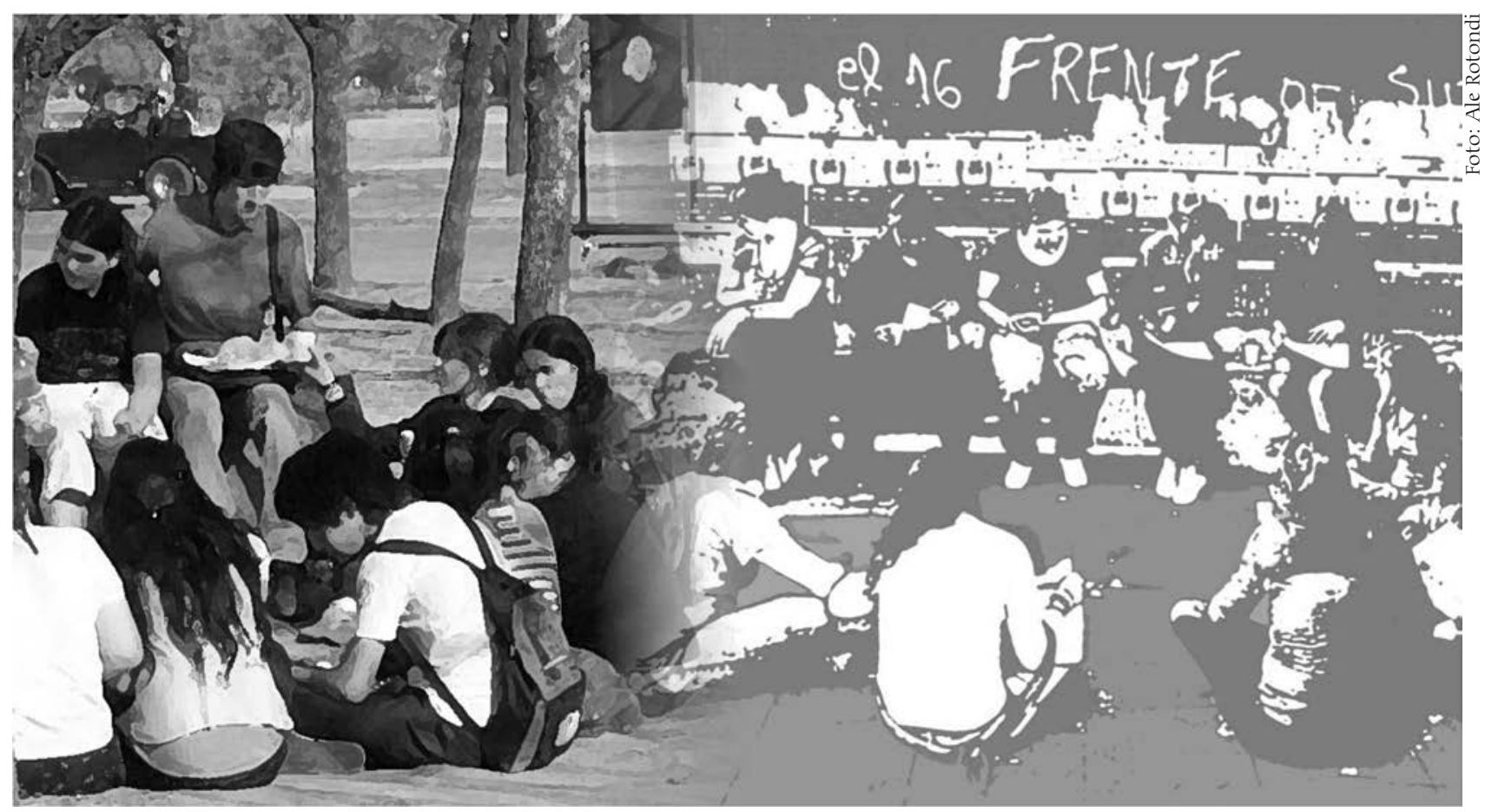

Conversaciones en el último encuentro de jóvenes de escuela secundaria 2010

\section{Introducción}

El estudio de los derechos gremiales de los estudiantes secundarios en las escuelas públicas ha sido una temática escasamente indagada en nuestro medio y pareciera que solo es objetivada ante situaciones de conflicto, como por ejemplo las tomas de escuelas del 2010 en Buenos Aires y Córdoba. Por otra parte, las dificultades organizativas planteadas por los estudiantes secundarios en esas instancias, se encuentran relacionadas con diversos aspectos de sus propias trayectorias estudiantiles de participación, en centros de estudiantes, en las estructuras de poder institucional y normativo instaladas en las escuelas, y en otras organizaciones escolares. El interjuego de los diversos sujetos institucionales por motivos gremiales, se complejiza con la dinámica y ejercicio del poder en la escena institucional escolar. Ante esta cuestión surgen diversos tipos de interrogantes. ¿Es posible fortalecer el proyecto institucional en la escuela pública involucrando a los estudiantes en su dimensión gremial? ¿Qué parámetros organizativos se plantean respecto de la participación ciudadana de los jóvenes estudiantes? ¿Qué viabilidad plantean los gérmenes organizativos de estudiantes secundarios en los formatos actuales? ¿Qué aporta el Trabajo Social en este tipo de instancias?

Teniendo en cuenta los rasgos institucionales de las escuelas públicas de Córdoba intentaremos entonces, reflexionar sobre los aspectos organizativos 
${ }^{1}$ Investigación desarrollada en la Escuela de Trabajo Social, Universidad $\mathrm{Na}$ cional de Córdoba con el apoyo de la Secretaría de Ciencia y Técnica UNC. 20010 - 2011. Dirigida por Mgtr. Gabriela Rotondi, Investigadores principales.

LIc. Cristina Fonseca y Lic. Dolores Verón. Investigadores Auxiliares. Lic. Marcelo Corona, Lic. Adriana Varela; Lic. Elizabeth Barraza, Lic. Paula Gaitán${ }^{2}$ ARTÍ́CULO 4‥- El Estado Nacional, las Provincias y la Ciudad Autónoma de Buenos Aires tienen la responsabilidad principal e indelegable de proveer una educación integral, permanente y de calidad para todos/as los/as habitantes de la Nación, garantizando la igualdad, gratuidad y equidad en el ejercicio de este derecho, con la participación de las organizaciones sociales y las familias. de los estudiantes secundarios, a partir del estudio de los procesos de agremiación al interior de un espacio institucional educativo: las escuelas públicas y su aporte a la condición ciudadana de los estudiantes secundarios. Consideraremos particularmente como marco, aspectos normativos propios de la política educativa respecto de la participación juvenil en nuestro medio (Legislación nacional y normativa de centros de estudiantes Res. 124/10 del Gobierno Provincial de Córdoba).

\section{Derechos ciudadanos, derechos gremiales}

Los derechos ciudadanos, sean civiles, políticos, sociales gremiales o reproductivos permanecen condicionados en términos estructurales, por la lógicas de organización social tanto como por los espacios sociales e institucionales. Además, los procesos mediante los cuales se instalan socialmente las instancias organizativas no son objetivados fácilmente. En tal sentido, la investigación "Agremiación y la acción juvenil: un derecho ciudadano que forma ciudadanos/as" ${ }^{11}$ busca aportar al estudio de los procesos de institucionalización de centros de estudiantes en escuelas medias y su relación con la construcción de representaciones sociales de los jóvenes en torno a la ciudadanía. Esto nos lleva a hacer un anclaje inicial en la noción de Agremiación Juvenil. Esta noción -en los ámbitos educativos- plantea ciertas novedades en lo que hace a los aspectos normativos en nuestro medio (Córdoba - Argentina) En el campo de la educación, desde la ley 26.206 de Educación Nacional se habilita desde derechos y obligaciones a los alumnos, a conformar organización desde la escuela. Esta norma, se inscribe en el escenario de las políticas públicas educativas y convoca al conjunto de docentes, alumnos, intelectuales, familias, funcionarios, académicos, dirigentes políticos y sociales, líderes culturales y religiosos, con el objeto de construir una política educativa signada por la democracia pedagógica de un Estado Constitucional. Desde este marco, se estarían posibilitando algunas condiciones para la construcción ciudadana de los jóvenes secundarios desde el ángulo gremial. Esto al menos en términos teóricos - formales de la implementación de la política pública.

Particularmente se apunta en el Artículo 4 de la norma², algunos elementos que inscriben la participación como posibilidad de las organizaciones sociales y las familias. Así como también el Artículo 8, apunta respecto de los marcos valorativos a impulsar en la formación: "La educación brindará las oportunidades necesarias para desarrollar y fortalecer la formación integral de las personas a lo largo de toda la vida y promover en cada educando/a la capacidad 
de definir su proyecto de vida, basado en los valores de libertad, paz, solidaridad, igualdad, respeto a la diversidad, justicia, responsabilidad y bien común".

Se incluyen además en el artículo 11, fines y objetivos de la política entre los que recuperamos particularmente el punto "b del artículo 11" que plantea la posibilidad de: Garantizar una educación integral que desarrolle todas las dimensiones de la persona y habilite tanto para el desempeño social y laboral, como para el acceso a estudios superiores; brindar una formación ciudadana comprometida con los valores éticos y democráticos de participación, libertad, solidaridad, resolución pacífica de conflictos, respeto a los derechos humanos, responsabilidad, honestidad, valoración y preservación del patrimonio natural y cultural; garantizar, en el ámbito educativo, el respeto a los derechos de los/as niños/as y adolescentes establecidos en la Ley $\mathrm{N}^{\circ}$ 26.061. i) asegurar la participación democrática de docentes, familias y estudiantes en las instituciones educativas de todos los niveles". Texto de la norma citada.

Estos objetivos que pueden constituir un marco de participación, son elementos aún imprecisos para aludir a la idea de agremiación juvenil. Motivo por el cual consideramos necesario recuperar particularmente una normativa provincial, la Res. Min 124 (10/11/2010), la cual plantea en su artículo 1: "Autorizar la constitución y funcionamiento de un órgano de representación estudiantil bajo la denominación Centro de Estudiantes en cada una de las instituciones educativas de gestión estatal dependientes de la Dirección General de Educación Media de la Dirección General de Educación Técnica y Formación Profesional; de la Dirección General de Educación Superior; de la Dirección de Regímenes Especiales; y de la Dirección de Jóvenes y Adultos". Desde esta normativa ministerial, se propone entonces al Centro de Estudiantes como el único órgano de representación de los estudiantes regulares matriculados en el establecimiento. En su art. 3 la norma reconoce además, como principios generales, los siguientes derechos, garantías y obligaciones recíprocas: Participación libre, plena y democrática de todos los estudiantes del establecimiento; elegir y ser elegidos; Voto universal y secreto; plazos expresos y renovación de los mandatos ejecutivos; derecho a peticionar; derecho a conocer sobre el funcionamiento del Centro de Estudiantes y sus finanzas; publicidad de las resoluciones, actos y acciones de los cuerpos orgánicos; rendición de cuentas ante la Asamblea General; representación de las minorías, cuando estas superen el 20 \% de los votos validos emitidos en la elección respectiva. La norma 124 instala además, funciones particulares para estas instancias organizativas las cuales se 
3 Se han realizado a la fecha cuatro encuentros de centros de estudiantes, con la participación de más de 4000 jóvenes de escuelas públicas prioritariamente, organizados por la Cátedra Teoría, Espacios y Estrategias de Intervención IV Institucional desde la línea de extensión del equipo. Esta experiencia y sus an-
tecedentes han sido premiados en diversas ocasiones obteniendo fondos para su desarrollo del programa de Mejora de la escuela media, Ministerio de Eduación de la Nación (2004/2007); Facultad Latinoamericana de Ciencias Sociales Flacso (2007); Voluntariado Universitario Nacional (2009), y reunió a diversos sectores docentes, estudiantiles y egresados universitarios que colaboraron e hicieron posible la experiencia.

mencionan como: ejercer la representación de los estudiantes; peticionar y gestionar ante el equipo directivo las demandas estudiantiles; promover el claustro estudiantil; promover acciones tendientes a la resolución pacífica de conflictos; promover entre los estudiantes y miembros de la institución el uso adecuado y mantenimientos de los bienes materiales y culturales presentes en el establecimiento; participar de los espacios pertinentes; colaborar a que se forme un clima de estudio apropiado al cumplimientos de pautas y metas, instalando de esta manera las posibilidades de acción gremial estudiantil en el marco de una norma acotada pero concreta.

En este contexto, la escuela se plantea como un ámbito en el que el encuentro de los jóvenes es una instancia viable para instalar, aludir y desarrollar derechos gremiales. Donde sin embargo se requiere instalar acciones concretas relativas a la organización de un grupo del establecimiento escolar y procesos de apropiación de determinados acuerdos, roles, pautas en el ejercicio del poder, en los diversos grados de liderazgo donde se plantee la promoción de estrategias de relaciones organizadas en función de un objetivo común que resulta ser en definitiva el para qué del grupo.

\section{Historias y contextos de participación: ¿qué plantean los jóvenes?}

Las historias de participación de los jóvenes cordobeses en los últimos años recorren instancias que los vinculan a ámbitos de la cultura, ámbitos educativos, prácticas sociales diversas e instituciones de la política pública, entre otros. Sin embargo se destacan en los últimos cinco años en nuestra ciudad de Córdoba espacios de participación que se relacionan con el encuentro y el compartir ámbitos de acción, decisión y expresión política, como es el caso de prácticas vinculadas a la agremiación juvenil que se expresa en los Encuentros de Centros de Estudiantes Secundarios, realizados desde la Universidad Nacional de Córdoba ${ }^{3}$, y las tomas estudiantiles de escuelas ocurridas durante 2010.

Estos espacios despliegan particularmente la posibilidad de estudiar las maneras en que los jóvenes instalan posibilidades de agremiación en ámbitos institucionales, con lógicas de mayor formalidad y con marcos que si bien abren el juego a la oportunidad organizativa de los jóvenes, simultáneamente obturan estas mismas posibilidades con otras normativas y/o discursos que plantean el cierre a los canales de participación en las escuelas. Estas prácticas y en particular los encuentros de centros de estudiantes, en los cuales el debate de 
la agremiación ha sido el eje que motoriza a los estudiantes secundarios, junto a una demanda social construida con las tomas estudiantiles nos plantea algunos datos relativos a lo que los jóvenes piensan respecto de la agremiación juvenil.

En primera instancia entonces queremos recuperar algunos elementos derivados de las conclusiones de los encuentros de Centros de Estudiantes. Particularmente recuperar elementos respecto del interrogante: ¿Qué plantean los estudiantes secundarios cuando se les pregunta sobre: ¿qué es un Centro de Estudiantes? ${ }^{4}$.

Al respecto se expresa respecto de los Centros de Estudiantes en los encuentros: "Son el lugar donde se plantean dudas y se realizan ideas", "nuestro lugar"; "Para hacer frente a los "profes" y directivos"; "Trabajo en conjunto"; "El Centro de Estudiantes es un conjunto de personas que se reúnen para un fin que es el bien común"; "Expresar lo que se piensa"; "Colaborar"

Esta diversidad aporta respecto de las miradas de la cuestión organizativa, y obviamente no encontramos en el colectivo estudiantil elementos que hagan una lectura única o compacta respecto de lo que significa organizarse. Participar y organizar aparecen como caras de una misma cuestión, que además expresan objetivos diversos, que son en definitiva lo planteado por los jóvenes cuando se pregunta qué es un Centro de Estudiantes. Es claro que prima la idea de lo colectivo como elemento que los aglutina en un Centro de Estudiantes así como también la idea de comunicar y expresar, cuestiones propias. Esto se repite además en las entrevistas realizadas desde la investigación que reflejan algunos particulares rasgos de la organización estudiantil expresados en los siguientes testimonios: "El Centro de Estudiantes, es un medio de comunicación que hay entre los directivos mayormente, y los profesores; entre los alumnos es el medio que nos comunica más directamente, entre lo que es realidad del alumnado y los directores, a los Inspectores de los Colegios, los chicos nos dan sus quejas, nosotros lo ponemos en nota, o se lo pasamos oralmente a Cecilia, o leemos notas, de lo que hagamos depende nuestro éxito, si tenemos algún problema, tratamos de solucionarlo con los profesores, que hay veces mal entendidos entre los alumnos con los profesores, a veces mal entendido entre los alumnos con los preceptores de modo que sean equitativamente y que las cosas no sean injustas, hay veces que hay profesores o profesoras que vienen con un mal día...y se la agarran con nosotros y esos son días de discusiones que siempre pasan. El fin del Centro de Estudiantes es esa comunicación que existe entre los alumnos y los profesores para solucionar los malos entendidos y problemas que hay".

4 Nos basaremos en los testimonios de alumnos entrevistados en la investigación pertenecientes a las escuelas de la Escuela Jerónimo Luis de Cabrera y Carolina Visca. Córdoba 2010. 
"Aparte trabajar por nuestros derechos, también hacer el Colegio menos aburrido en sí, plantear actividades como campeonatos, como el tema del mete gol, la fecha de la primavera, partes de trabajo en gimnasio..."

"Hay que tener en cuenta que son relaciones humanas, que estamos hablando de personas y por más que seamos Centro de Estudiante nosotros somos alumnos también, y no tenemos por ahí la autoridad como para ir y cuestionar y solucionar problemas que están fuera de nuestro alcance. Hay cosas que muchas veces nos piden y que nosotros no podemos ir a solucionar, que están fuera del Centro de Estudiante..." "Yo creo que el hecho de que se llame Centro de Estudiantes es porque está liderado por estudiantes, y me parece que es importante que cada curso tenga su líder o representante ante la colectividad de la institución. Me parece que el centro es algo como la minoría de los alumnos en conjunto, y sirve un montón para la hora de manifestarnos nosotros, en lugar de ir todo el curso a hablar sobre tales puntos, sino que un representante hable con la directora en nombre de todos..." La participación yo creo que es escuchar y ser escuchado. Me parece que es comunicarse, requiere de otras personas, uno no puede participar de algo si está solo, también necesita de una organización"

"Yo creo que participar en cualquier ámbito no solo nos podemos referir a un Centro de Estudiante. Yo creo que el hecho de levantarse y hablar con los compañeros del curso ya es un modo de participación, opinar sobre algo es participar. Manifestarse artísticamente es participar. Es un concepto amplio en el que uno puede referirse distintos tipos de manifestaciones".

Estos elementos derivados de las entrevistas cualitativas de la investigación, señalan algunas cuestiones significativas respecto de los procesos de conformación de un Centro de Estudiantes:

- En primera instancia el hecho de reconocerse entre iguales a la hora de la formulación de una organización. Ser iguales en una condición, la de estudiantes que plantea problemáticas comunes y realidades comunes que requieren en ocasiones "ser comunicadas" y donde pareciera que existe la posibilidad de construir "interlocutores" o "interlocución" con otros actores escolares. Esto creemos que implica agremiación.

- En segundo lugar esta interlocución implica poder expresar "cosas del colectivo", cuestiones comunes que requieren ser tratadas por algún motivo (dificultades escolares, reclamo de derechos, puesta en común).

- En tercer lugar pareciera que emerge una necesidad de participación vinculada también a las posibilidades de hacerse escuchar como colectivo 
que acciona ante sus propios problemas de agremiación.

- Y en cuarto lugar no queremos dejar de mencionar que desde lo disciplinar planteamos la existencia de una nueva demanda profesional que se plantea desde la institución escuela para el abordaje, apoyo y fortalecimiento a una organización estudiantil.

Se complementan estos testimonios con las expresiones respecto de aquello a lo que aluden los estudiantes cuando se les interroga sobre los para qué de los Centros de Estudiantes. En los encuentros aparecen como respuestas del por qué organizarse: "Permite el ejercicio de la capacidad de asociarse"; "Armar iniciativas"; "Escuchar y aportar"; "Mejorar la calidad de vida en la escuela (académica, infraestructura, etc.)"; "No solo es un espacio para quejas, sino para iniciarse en la experiencia democrática"; "Plantear proyectos"; "Respaldo"; "Acompañamiento"; "Pertenencia".

Estas expresiones puntuales que señalan las aspiraciones de los jóvenes estudiantes, tienen su correlato en las demandas que las instituciones realizan, concretamente a la cátedra Teoría, Espacios y Estrategias de Intervención IV - Institucional y Trabajo Social V Intervención en Instituciones, de la Escuela de Trabajo Social, Universidad Nacional de Córdoba ${ }^{5}$, cuando plantean pedidos de intervención directa para apoyar estas organizaciones.

\section{Al respecto podemos señalar lo siguiente:}

- Se plantean desde 2006 demandas relativas a formación y/o fortalecimiento de Centros de Estudiantes que se expresan como: "crear o conformar un Centro de Estudiantes"; "necesidad de acompañamiento al Centro de Estudiantes"; "necesidad de apoyar el fortalecimiento del centro"; "ayudar a conformar el centro"; "facilitar procesos de comunicación desde el Centro de Estudiantes"; "trabajar sobre identidad y participación juvenil"; "aportar al mejoramiento de los vínculos entre los actores institucionales y la concientización y armado del centro de Estudiantes" Estas demandas son formuladas por diversos actores institucionales que ven en el Trabajo Social la posibilidad de abordaje del asunto y que lo viabilizan en particular por la cátedra que realiza un abordaje institucional.

- Las demandas muestran que si bien pareciera que en la escuela está presente la normativa relativa a la conformación de Centros de Estudiantes y aun la necesidad de los mismos, no aparecen - al menos en las demandas relevadas-procesos de acción institucional previos a la conformación de

5 Todos los miembros del equipo de investigación pertenecen o se han formado en el marco de esta cátedra, del mismo modo que la experiencia de trabajo con los Centros de estudiantes ha tenido su inicio y desarrollo en este ámbito. Las demandas a las que aludimos la realizan instituciones escolares que desean contar con equipos de ésta cátedra para realizar trabajos anuales entre estudiantes y docentes. 
Centros de Estudiantes, por lo que la expectativa vincula la posibilidad de participación a acciones específicas de Trabajo Social. O en los casos donde si existe antecedente la demanda se centra en fortalecer canales de encuentro, diálogo, etc. entre la institución y el Centro de Estudiantes.

- Si está claro, que los desarrollos organizativos aparecen aun débiles y cuenta de ellos dan las demandas que realizan docentes o directivos sobre el tema, y no necesariamente son realizadas por los estudiantes.

\section{Podemos dejar constancia que:}

- Demandan en 2006 a la cátedra 53 escuelas, de las cuales solo dos expresan claramente una demanda para apoyar la conformación de centros de estudiantes.

- Durante 2007 demanda a la cátedra apoyo para la conformación y apoyo a Centros de Estudiantes en tres casos sobre 19 demandas recibidas por la cátedra.

- Durante 2008, demandan a la cátedra 23 escuelas, de las cuales siete solicitan atención para el Centro de Estudiantes.

- En 2009 demandan a la cátedra 30 escuelas, de las cuales siete corresponden a pedidos de apoyo para la conformación o seguimiento del Centro de Estudiantes de las escuelas.

- En 2010 sobre 30 demandas diez -un tercio de ellas- solicitan apoyo, o aporte al fortalecimiento de Centros de Estudiantes. Podemos observar un crecimiento de la demanda para apoyo a organizaciones estudiantiles que podemos relacionar con al menos tres cuestiones: la convocatoria en sí que realiza la cátedra; las normativas existentes y sus modificaciones y el impacto de los Encuentros de Centros de Estudiantes que definen no solo una progresión respecto de la participación; sino además una divulgación y con ella requerimientos sobre el tema.

Otro elemento que podemos leer de la expresión de estas demandas son las diferentes posiciones de los adultos ante el tema: Crear o conformar un centro, sin duda es tarea de sus principales actores, los estudiantes. La demanda de crear, conlleva, o al menos eso pareciera, una iniciativa de actores institucionales que tal vez consideren necesario dar el puntapié inicial en estos procesos. ¿Qué implica esto para las organizaciones juveniles? ¿Podemos pensar en adultos llevando la delantera organizativa en un espacio como la escuela que además conducen los mismos adultos? Sin duda es un tema de reflexión. Por otra parte emerge de las expresiones de las demandas otra cuestión, el hecho de que 
las organizaciones estudiantiles se encuentran en diverso tipo de procesos de gestión organizativa, donde no tiene la misma relevancia crear, que apoyar, o fortalecer. Estos matices expresan estas posibles diferencias en los procesos de organización de los estudiantes.

Podemos aun leer otro elemento de estas demandas, y es el hecho de las problemáticas colaterales que expresan los adultos cercanas a lo organizativo, la comunicación; el abordaje de conflictos, pareciera que son una vía de entrada al tema -al menos para los adultos- que también hay que considerar a la hora de relevar experiencias.

\section{Centros de Estudiantes e institucionalización.}

La institucionalización en tanto proceso deja huellas en la estructura, dinámica, en las prácticas, logros y fracasos en la institución, en los conflictos y obviamente en la cultura de cada organización. Por otra parte el proceso de institucionalización, enmascara el hecho de que la construcción de las instituciones no se opera en el vacío, sino que existen elementos condicionantes desde lo estructural, desde los contextos sociales, históricos, políticos, entre otros. El estudio del proceso de institucionalización- en este caso de organizaciones juveniles surgidas en espacios escolares - plantea particulares desafíos que se vinculan a las huellas que plasman las instituciones en los sujetos. Estudiar el proceso de institucionalización de Centros de Estudiantes de escuelas medias y su relación con las representaciones sociales de los estudiantes en torno a la ciudadanía es algo para nosotros por demás significativo. ${ }^{6}$ Precisando ya las necesidades en relación a la conformación de organizaciones y las posibilidades de participación, organización y agremiación juvenil, surgen a nivel de la investigación y de la experiencia que desarrollamos cuatro cuestiones relevantes:

En primera instancia y reiterada en los cuatro años de trabajo en los encuentros de centros de estudiantes, surge la necesidad de aportes en lo organizativo gremial, el entrenamiento en formas de participación y la necesidad -de los jóvenes- por conformarse como centros de estudiantes. Esta es tal vez la necesidad que opera con mayor fuerza. Sin embargo relevamos además tres cuestiones que constituyen demandas de abordaje de parte de los estudiantes y que son: la necesidad de abordar la problemática de la violencia en el noviazgo; la necesidad de abordar la implementación de la ley de educación sexual; y la necesidad de abordar los derechos ambientales. Estas temáticas surgen desde

${ }^{6}$ Rotondi, Gabriela, Proyecto de Investigación: Género y Educación Sexual: Institucionalización de una política pública. (En el marco de la implementación de la ley 26150, Programa de Educación Sexual en las Escuelas)
cond Programa de Educa- 
${ }^{7}$ Encuentro Regional realizado en la Ciudad de Oliva por los jóvenes como por los profesionales que identifican estas necesidades en las diversas instancias diagnósticas. En este sentido el hilo conductor que las vincula es la necesidad de acceso a derechos particulares, tanto de agremiación, como de una vida sin violencia, derecho a la educación sexual y derechos ambientales. Más tardíamente y en una instancia regional ${ }^{7}$ surge la necesidad de abordaje de las relaciones conflictivas con las sustancias y el alcohol. Este tipo de demandas ponen foco en la intervención de Trabajo Social en torno a las representaciones sociales y las necesidades organizativas, particularmente. En este sentido vemos que aun reconociendo las historias disciplinares y la ausencia histórica de demanda solvente al Trabajo Social, a cambio de sus servicios, la demanda mediada por estas instituciones, ofrece un punto de apertura a las tradicionales pautas de sujeción a los mandatos institucionales definiendo desde la demanda un punto de autonomía para el ejercicio profesional que se valida también en esa demanda. Si bien la autonomía de la disciplina es derivada de las correlaciones de fuerzas construidas en un campo profesional, que históricamente ha desarrollado esa pauta profesional, esto tampoco podemos plantearlo como una mera instancia de acción profesional, y queremos entonces registrar este antecedente de cinco años, donde instituciones públicas establecen particulares tipos de demanda de acción a un Trabajo Social que presuponen puede abordarlas. Cabe señalar que desde esta cátedra se trabaja con modalidad de demandas desde el año 1994.

Por otra parte y ya volviendo a la posibilidad de abordar el estudio de los procesos de institucionalización de estas organizaciones, ligado a las representaciones sociales y el entrenamiento ciudadano que propician estas instancias organizativas, queremos señalar algunos elementos particulares de la lectura de esta cuestión. Los procesos de democratización en orden a la ciudadanía en las instituciones, tienen relación con diverso tipo de dimensiones presentes en la vida institucional tales como la dimensión organizativo normativa; cultura; comunicación; el proyecto institucional; entre otras: Pero además estos procesos se vinculan a las posibilidades que los sujetos tienen de conformar y dar desarrollo a sus organizaciones de acuerdo a sus propias necesidades en el seno de las instituciones. Este hecho cuenta para nosotros como uno de los disparadores de las demandas de acción profesional. En relación a esto además, es significativo para nosotros recuperar la noción de necesidad planteada por Nancy Fraser (1991) respecto de "las políticas de interpretación de las necesidades" donde entendemos que la autora provoca

Palobra No. 12. Agosto de 2010 - Julio de 2011 
una ruptura respecto de la relación necesidad - satisfactor y plantea que las respuestas políticas a las necesidades de los sujetos no obedecen solamente a una relación con los recursos disponibles, sino que considera el discurso de las necesidades como un espacio de contienda donde los grupos con recursos discursivos (y no discursivos) desiguales compiten por establecer como hegemónica sus interpretaciones respectivas sobre lo que son las legítimas necesidades sociales Se trata de una lucha por las necesidades comprendidas en términos analíticos por tres momentos: la disputa por otorgar estatuto público; la lucha por la interpretación y la lucha final por la satisfacción. Es claro que la necesidad de organización estudiantil en este ámbito es un asunto que se empieza a debatir en los espacios públicos fundamentalmente en los últimos años y en tal sentido, la conformación de organizaciones juveniles en la Escuela, involucra una lectura de la lucha de las necesidades; sus procesos de desarrollo y fortalecimiento institucional, y el estudio de los procesos de representación que contribuyen a la construcción de prácticas, valores y representaciones en torno a los derechos y la ciudadanía entre los jóvenes. "El acto de institución es un acto de comunicación, pero de un tipo particular: significa a alguien su identidad, pero a la vez en el sentido que la expresa y la impone expresándola frente a todo..." "Instituir, dar una definición social, una identidad, es también imponer límites." Bourdieu (1985 pag:81) En este sentido el estudio del proceso de institucionalización - en este caso de organizaciones juveniles surgidas en espacios escolares - plantea particulares desafíos que se vincularán a las huellas que plasman las instituciones, en los sujetos. Pero además y cuando aludimos a huellas tendremos que inventariar y analizar los procesos políticos por los cuales circulan las oportunidades organizativas de los jóvenes. Hay que considerar además que se trata de procesos que desarrollan al interior de instituciones - organizaciones y en ese sentido el pedido de ayuda - demanda está de alguna manera garantizado por ese respaldo institucional que se le ofrece desde la escuela a los Centros de Estudiantes.

Las cuestiones abordadas con los jóvenes en las instancias organizativo/ políticas de los Centros de Estudiantes definen un primer foco de articulación en orden a la condición y formación ciudadana. En la participación estudiantil se plantean particularidades. Diversas podrían ser las formas de planteamiento, descripción y/o explicación de la participación estudiantil de los secundarios; tanto como acción individual; derecho durante las instancias formativas; la mirada de la participación juvenil desde identidades sociales particulares; la participación desde, o, en la política y/o espacios públicos. Sin embargo es 
también necesaria la lectura de las instancias de participación política desde una posición y conflicto de intereses de esa situación y posición particular: la de estudiantes secundarios que operan en un espacio organizativo, los Centros de Estudiantes, cuyo contexto es la institución escuela. Los procesos de participación en las instituciones educativas, plantean además relación con dimensiones analíticas presentes en la vida institucional tales como la dimensión organizativa normativa; la historia y cultura institucional; las pautas de comunicación; el proyecto institucional Garay, L (1994); la comunicación y sus discursos, la circulación del poder, los procesos de institucionalización Berger y Luckman (1968) y los ritos de institución Bourdieu (1985), entre otras. Pero además si se trata de estudiar organizaciones presentes en las instituciones, estos procesos plantearán otras complejidades, vinculaciones por ejemplo con las posibilidades que los sujetos tienen de conformar y dar desarrollo a sus propias organizaciones, de acuerdo a su propia lectura de sus necesidades en el seno de las instituciones. Cuestión que de por si plantea una primera dificultad, la de estudiar organizaciones sociales en el seno de instituciones -como la escuela- cuyas misión puede validar o contraponerse a las definiciones de las organizaciones estudiantiles.

El abordaje involucra la Participación y Organización de los estudiantes; y simultáneamente problemáticas que tienen su propuesta de abordaje en espacios públicos. Estas cuestiones abordan una particular noción de derechos, y entrenamiento colectivo en ámbitos de acción pública, abordando la titularidad y el ejercicio ciudadano. Aún sigue siendo rígida, desde algunos espacios, la apertura a la participación de adolescentes, suele considerarse un ámbito sin autoridad y sin chance de decisiones, planteadas como desafiantes y transgresores de normativas, rebeldes. Esta visión obviamente, que no compartimos, aleja de los debates y entrenamientos de su condición ciudadana a los jóvenes. Es fundamental apostar entonces a la promoción de espacios de encuentro donde jóvenes pueden discutir junto a otros jóvenes cuestiones que conforman su dinámica cotidiana y se convierte en signos de preguntas cuando desde las autoridades son pasadas por alto o no valoradas con la relevancia que deberían serlo.

Las dificultades encontradas en los estudios y experiencias previas pareciera que tienen relación con la posibilidad de instalar en la trama institucional de la escuela una organización estudiantil de carácter gremial. Nos interrogamos entonces respecto de estos procesos de conformación y/o consolidación de organizaciones juveniles en el ámbito escolar como Centros de Estudiantes; 
las acciones y problemas que los fundamentan, las normativas que los avalan, las articulaciones posibles. Nos interesa estudiar los procesos de institucionalización de estas organizaciones, en el marco de las acciones que los jóvenes realizan como pautas de entrenamiento de su condición ciudadana ya que consideramos que las prácticas desarrolladas en organizaciones gremiales estudiantiles podrían aportar en orden a las representaciones sociales relacionadas con la condición ciudadana de los sujetos. Y al respecto observamos además que aun no han sido exhaustivamente relevados los procesos de constitución de organizaciones gremiales estudiantiles en el ámbito institucional de la escuela media salvo en los casos citados y por ende aun no podemos arriesgar conclusiones apresuradas. Esta cuestión demanda la necesidad de identificar y hacer visibles acciones que contribuyen al protagonismo juvenil y el desarrollo de la condición ciudadana en los ámbitos escolares teniendo en cuenta además las nociones planteadas de: demanda social a los profesionales de Trabajo Social; y el cruce que plantea la existencia de una organización al interior de una institución - organización en la que se juegan procesos y poderes de manera simultánea. Observamos además que algunas de las dificultades organizativas se encuentran relacionadas con las estructuras de poder institucional, en el caso particular de los escenarios escolares, el interjuego se complejiza por la dinámica de los diversos actores y el ejercicio de poder que cada uno hace -donde los estudiantes son actores con relativo poder institucional asociados a un profesional que tampoco cuenta con una validación social que lo empodere- poniendo en evidencia la complejidad de las relaciones en el campo abordado. En este transcurso de cuestiones la realidad organizativa de los estudiantes cobra la fuerza necesaria que impulsa a la gestión de acciones con eje en la dimensión de la participación y la construcción ciudadana entre los jóvenes. Los derechos ciudadanos -sean civiles, políticos, sociales o reproductivos- permanecen condicionados en términos estructurales, por la lógicas de organización social capitalista, tanto como por los espacios que resultan cada vez más estrechos para su ejercicio efectivo en el marco social y político. Es en la práctica de todos los días, en sus relaciones, en la vida cotidiana, donde se construye la credibilidad y la confianza de los ciudadanos/as. Este hecho trae aparejado entonces la necesaria búsqueda de posibilidades de la escuela de transformarse en una de las instituciones viables para la formación y el fortalecimiento de la ciudadanía. Nos enfrentamos, en las sociedades latinoamericanas, a la paradoja de sociedades democráticas con ciudadanos nominales o ciudadanos incompletos, es decir, con falsos ciudadanos o con ciudadanos que no pueden ejercer plenamente los atributos correspondientes a esa condición. Sin duda, 
el análisis de este problema conecta, en una trama conceptual compleja, las categorías de ciudadanía, democracia, igualdad y derecho. La democracia presupone, pues, ciudadanos iguales en términos de sus derechos, y la noción de ciudadanía no se entiende sin un sistema de derechos. Y entre ellos los derechos sociales, como la educación son tal vez una clave para esa constitución ciudadana de los sectores juveniles, si operan como instancias de entrenamiento de las prácticas ciudadanas y de los procesos de formación de las representaciones sociales en orden al tema, y en este caso en orden al aprendizaje de derechos gremiales.

\section{Conclusiones}

Es complejo para nosotros ofrecer conclusiones definitivas, en primera instancia porque estamos aludiendo a una investigación y experiencia en curso, y por otra parte porque pretendemos dejar planteados algunos mojones de nuestro debate respecto del ejercicio de Trabajo Social en el ámbito institucional.

Respecto de lo primero vemos necesario señalar la importancia que plantea el estudio de los procesos de institucionalización de las organizaciones estudiantiles atento a los procesos sociales - gremiales políticos planteados en nuestro medio en relación a un actor social que no solamente ha sido silenciado en diversos períodos históricos de nuestro país, sino que además es una actor al que suele remitírselo a acciones en el marco institucional de escaso poder organizativo o gremial, como pueden ser: "pinten la escuela" "limpien el patio", entre otros pedidos institucionales. En este sentido la novedad de demanda para el apoyo y fortalecimiento gremial de los estudiantes secundarios es una novedad en nuestro medio y en nuestra práctica profesional que abre el juego a una acción directa de agremiación y de debate y abordaje de las representaciones sociales en el tema.

Por otra parte reconocemos que las lecturas realizadas por los estudiantes respecto de la importancia de la agremiación para gestar nuevas formas de interlocución, planteada además en términos colectivos y tomando como eje los derechos que los vinculan a las prácticas ciudadanas, es una cuestión que consideramos motivo suficiente y necesario para desarrollar estrategias de trabajo, acompañamiento y acción junto a este sector emergente como actor social. 
Teniendo en cuenta además que en una realidad como la de Córdoba, transcurrieron muchos años para que el movimiento secundario estudiantil tuviera una normativa concreta (Res. 124 Ministerio de Educación de Córdoba) que avale su chance de agremiación; y un movimiento que accione ante problemáticas comunes, como fueran las tomas de escuelas durante 2010. Esto hace para nosotros relevante el estudio y su articulación con los debates de autonomía profesional en tanto implica que los Trabajadores/as sociales tomemos iniciativas respecto de demandas sociales efectuadas desde un ámbito particular -en este caso como la escuela-, respecto de cuestiones organizativas que pueden derivar en el abordaje de derechos concretos interviniendo tanto en la necesidad que de por si expresan como en las representaciones sociales y las relaciones sociales que en ese ámbito se construyen. Así como también en el mismo ámbito institucional que demanda y que puede abordarse en sus diversas dimensiones, organizativonormativa; cultura; estructura de poder; proyecto institucional entre otras.

\section{BIBLIOGRAFIA}

AQUIN, ACEVEDO, ROTONDI, CUSTO. Informes de investigación: Investigación: Jóvenes y adultos frente a la democracia. Análisis comparativos (2003); Vigencia de los valores de ciudadanía en la juventud cordobesa. Primera y segunda parte (estudio cualitativo y cuantitativo) (1998/1999). Estudios realizados en el marco del Programa de incentivos de la Universidad Nacional de Córdoba. Con subsidio de la Secretaría de Ciencia y Técnica. Dirección del Proyecto a cargo la Lic. Nora Aquín. Escuela de Trabajo Social. Universidad Nacional de Córdoba.

BERGER Y LUCKMAN. (1968) La construcción social de la realidad. Edit. Amorrortu Bs.As.1era. Edición.

BOURDIEU, P (1985) ¿Qué significa hablar? Economía de los intercambios lingüìsticos Ed. Akal S.A. Madrid.

FERNÁNDEZ, L (1994) Instituciones Educativas: Dinámicas Institucionales en situaciones críticas Edit. Paidós. Bs. As.

FRASER, Nancy (1991). La lucha por las necesidades. Esbozo de una teoría crítica, socialistafeminista de la cultura política del capitalismo tardío. En debates feministas, año 2, volumen 3. México.

GARAY, L (1994) Análisis institucional de las organizaciones educativas y sus organizaciones. Escuela de Ciencias de la educación.

QUIROGA, H (2001) Democracia y ciudadanía y el sueño del orden justo. En: Filosofías de la ciudadanía. HomoSapiens ediciones. Rosario. Argentina.

Resolución. 124/10 del Gobierno Provincial de Córdoba)

Ley 26.206 de Educación Nacional. Argentina, 21 de diciembre de 2006.

Palobra No. 12. Agosto de 2010 - Julio de 2011 